\title{
Brain Region Extraction Using MRI Image Processing To Aid Neurology System Of Patient
}

\author{
Vandana Jagdish Shah
}

\begin{abstract}
For patient presenting with symptoms of tumour should be diagnosed properly. Since treatment may not cure at the later stage, researchers must aim to produce maximal benefit to the patient with minimal burden, taking quality of survival into account as well as the duration. CT scan images are limited by the resolution of the imaging. In the field of medical resonance image (MRI) processing the image segmentation is an important and challenging problem in an image analysis. The main purpose of segmentation in MRI images is to diagnose the problems in the normal brain anatomy and to find the location of tumour. This paper proposes a novel algorithm for segmentation of MRI images to extract the exact area of the brain as preprocessing steps of location of the tumour. Neuroradiological research consists of several brain extraction algorithms which are useful for several post- automatic image processing operations like segmentation, registration and compression. The result of proposed algorithm is validated by comparing proposed algorithm with the results of the existing segmentation algorithm used for the same purpose.
\end{abstract}

Keywords-Image segmentation, clustering, k-means clustering, fuzzy c means clustering, brain extraction algorithm.

\section{Introduction}

The main reason of image segmentation is to partition an input image into meaningful regions with respect to our particular application. The major significance of applying segmentation technique is to obtain coarse and fine details of tissues of brain MRI images in detail (Atkins and Mackiewich, 1998). Image segmentation is mainly used to detect objects and boundaries like lines, curves, edges etc. Image segmentation is the process of assigning a label to every pixel in an image which then assigned the same label who shares the same particular characteristics. The result of image segmentation is a set of segments that collectively cover the interested region in an image, or a set of contours extracted from the image (Gonzalez and Richard, 2007). Each of the pixels in a region is similar with respect to some characteristic or computed property, such as color, intensity, or texture. Adjacent regions are significantly different with respect to the same characteristic.

The success or failure of computerized analysis procedure is specified by segmentation accuracy. The resulting contours after image segmentation of MRI images can be used to create 3D (Raya, 1990) reconstructions with the help of interpolation algorithms.

Vandana Jagdish Shah

Assistant Professor, Electronics and Communication Department Sarvajanik College of engineering and Technology India
Major applications of medical image processing are: detect and locate tumours and other pathologies issues (Brummer and Mersereau, 1993) measure tissue volumes, diagnosis, study of anatomical structure, surgery planning, virtual surgery simulation, and intra-surgery navigation. In general, there are three important aspects to be considered in MRI image segmentation. The first aspect is the speed of the algorithm. The segmentation of image should not consume much time. The second aspect is good edge connectivity of its segmenting result (Perona and Malik, 1990). The third aspect is good shape matching. Consequently, it will be reliable (Somasundaram and Kalaiselvi, 2011). The limited disadvantages of segmentations could be fatal problem, the computation time and over segmentation. The result is sensitive to the selection of the initial random centroids in some of the segmentation techniques. Some region segmentation techniques can produce blocky segments.

\section{Proposed Algorithm for Brain Region Extraction}

The main purpose of the flow chart shown in figure 1 is to detect the region of brain with many pre-processing steps. The input MRI image is in JPEG form which selects one of the slices from more than 100 slices of patient's data. That has to be T2 weighted image for fine analysis of the brain. Before finding tumour it is important to segment the region of interest. Over here the region of interest is only brain area which has to be removed from skull. 2D-BEA (2 Dimension Brain Extraction Algorithm) is one of the techniques which extract the brain area from skull and boundary. In 2D-BEA first of all in the first stage the background noise from the brain image will be removed using low pass filter and the output is further diffused to enhance the brain boundaries. This will be forwarded by thresholding in which mask for the coarse brain is generated. In the second stage, morphological based segmentation operation is performed with connected component analysis to extract the fine brain from the coarse brain portion obtained in first stage of 2DBEA.

As per the figure 1, the segmented brain region will be further given to 3D-BEA (3 Dimension Brain Extraction Algorithm) because the 2D-BEA has limitation for largest connected component identification (Somasundaram and Kalaiselvi, 2011). 3D-BEA may remove that limitation and could be implemented for further research. 2D-BEA is explained later in this paper. The method makes the use of geometric continuity of the brain region to extract the overall brain region. The method makes the use of following steps to extract the exact brain region in 3D-BEA.

Step 1: Initially MRI $\mathrm{T} 2$ - weighted image $\mathrm{t}(\mathrm{x}, \mathrm{y})$ is smoothed using Low Pass Filter (LPF) with a structuring element (STEL) as a morphological operation. LPF is given by (1). 
Proc. of the Intl. Conference on Advances in Information Processing and Communication Technology - IPCT 2016. Copyright (C) Institute of Research Engineers and Doctors. All rights reserved.

ISBN: 978-1-63248-099-6 doi: 10.15224/ 978-1-63248-099-6-27

$$
\mathrm{L}_{\mathrm{p}}(\mathrm{u}, \mathrm{v})=\mathrm{H}(\mathrm{u}, \mathrm{v}) \mathrm{T}(\mathrm{u}, \mathrm{v})
$$

Where, $T(u, v)$ shows the Fourier transform of input T2 weighted image $\mathrm{t}(\mathrm{u}, \mathrm{v}), \mathrm{H}(\mathrm{u}, \mathrm{v})$ is the transform function of LPF, the filtered image is obtained simply by taking inverse Fourier transform as shown in (2).

$$
\mathrm{I}(\mathrm{x}, \mathrm{y})=\operatorname{IFT}\left(\mathrm{L}_{\mathrm{p}}(\mathrm{u}, \mathrm{v})\right)
$$

Construction of LPF is given by (3).

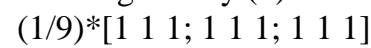

Here $3 * 3$ pixel size small filter has been used to remove the background noise.

Step 2: After above step the diffusion process is applied on filtered image for removing blurriness in the image. This process is calculating gradient in North, South, east and West direction using convolution mask as shown in below (4) to (7) respectively.

$$
\begin{aligned}
& \mathrm{hN}=\left[\begin{array}{lllllllll}
0 & 1 & 0 ; & 0 & -1 & 0 ; & 0 & 0 & 0
\end{array}\right]
\end{aligned}
$$

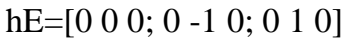

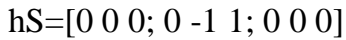

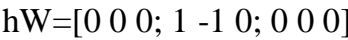

Step 3: Binarization of the diffused image I(x,y) has been generated using Riddler's thresholding method; where two means has been calculated. i.e. $\mu_{0}$ and $\mu_{1}$ are the means of each of the two components of the histogram separated by the initial value of threshold. This is calculated as shown in (8);

$$
\mathrm{T}(\mathrm{i})=\left(\mu_{0}+\mu_{1}\right) / 2
$$

Continuous iteration has been carried out to find the final threshold value using below equation.

$\mathrm{T}_{\text {final }}=$ tvalue; where tvalue is given by (9).

$$
\text { tvalue }=\mathrm{T}(\mathrm{i}) \text { for } \operatorname{abs}(\mathrm{T}(\mathrm{i})-\mathrm{T}(\mathrm{i}-1))>=1
$$

For final binarization if the pixel value is greater than tvalue then ' 1 ' is assigned to that pixel otherwise ' 0 ' is assigned to the same pixel.

Step 4: After getting binarized image using Riddler's threshold method the morphological operation has been performed to eliminate unwanted region. This uses a proper structuring element (STREL). The erosion has been processed for the same task using (10).

$$
\mathrm{I}_{1}=\mathrm{I}_{\mathrm{old}} \theta \mathrm{X}_{1}
$$

Where $I_{\text {old }}$ is the binarized image and $X_{1}$ is wide enough to detect the eye portion and detach the eyes with other small structure from the brain in axial scans. The final eroded image is $\mathrm{I}_{1}$. After this imopen command of MATLAB has been used to achieve image $I_{2}$ for removal of unwanted minute regions. This process is further gone through the dilation morphological operation using (11).

$$
\mathrm{I}_{3}=\mathrm{I}_{2} \oplus \mathrm{X}_{1}
$$

Where $I_{3}$ shows the dilated image with boundary strengthening which was lost during erosion. The brain area selection has been processed by finding Largest Connecting Component.

Step 5: If this area is greater compared to the one in the previous step we go for final extraction else we consider the $(\mathrm{X}-1)$ and $(\mathrm{X}+1)$ images both together and repeat the previous steps number 2 to 4 . The scalp-skull boundaries are very weak in T2-weighted images (Somasundaram and Kalaiselvi, 2011) and hence they are not preserved. In 2DBEA as shown in below figure 2, the diffusion process helps to compute an intensity threshold value automatically to segment the brain from non-brain tissues. Thus stage one is preserving brain borders. Thresholding helps to produce the rough mask brain. In the second stage main shape characteristics of object will be identified. Erosion and dilation produced the curve boundaries of brain regions of binary image.

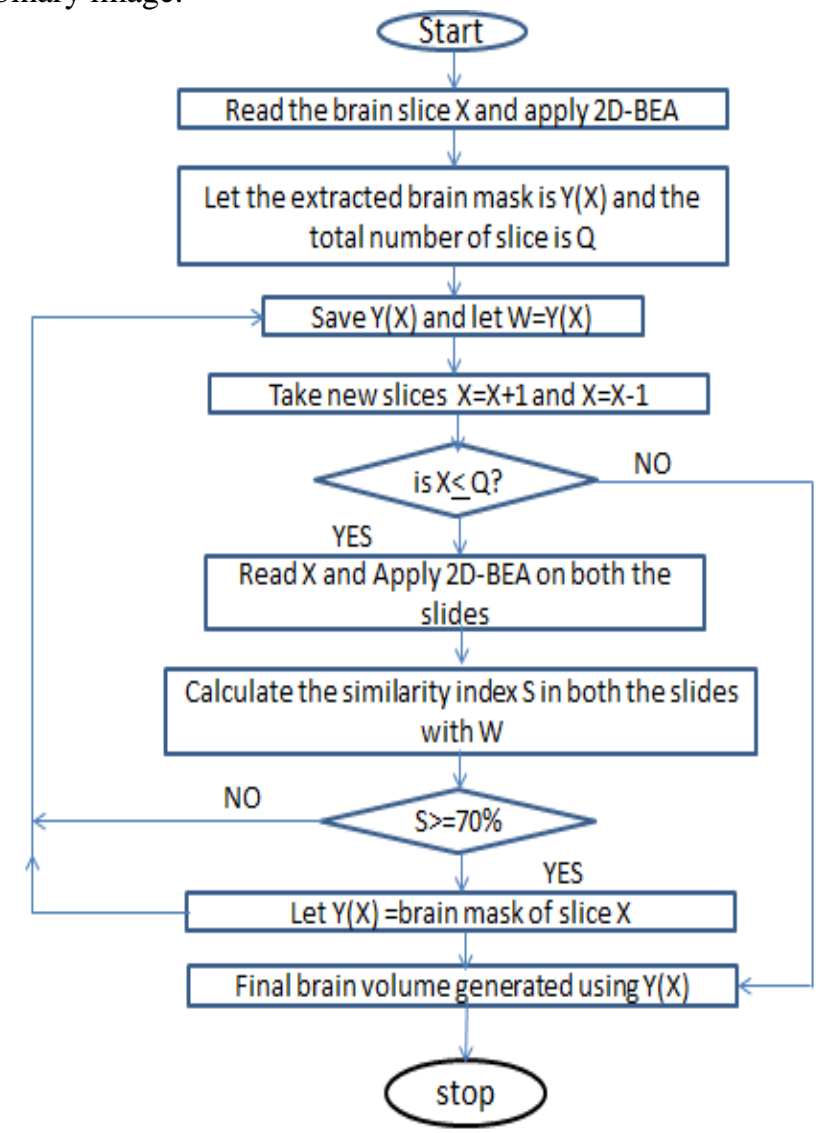

Figure 1. Proposed technique for Segmentation of T2 weighted brain MRI

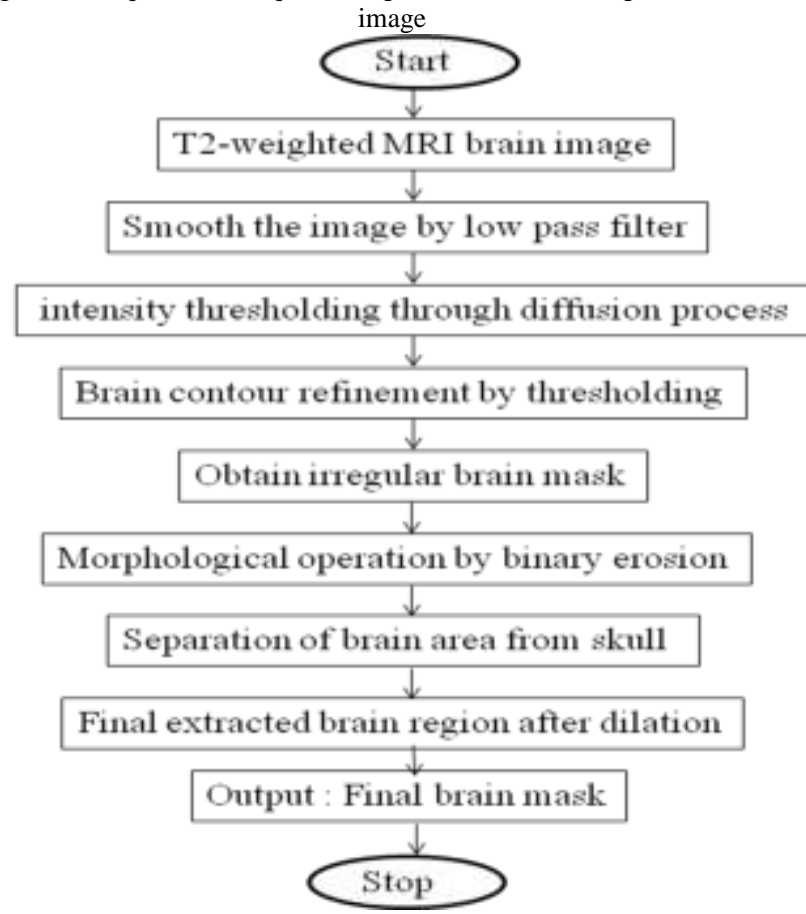

Figure 2. 2D-BEA algorithm for Segmentation of T2 weighted brain MRI image 
Proc. of the Intl. Conference on Advances in Information Processing and Communication Technology - IPCT 2016. Copyright (C) Institute of Research Engineers and Doctors. All rights reserved. ISBN: 978-1-63248-099-6 doi: 10.15224/ 978-1-63248-099-6-27

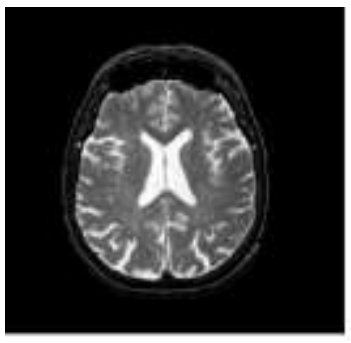

(a)

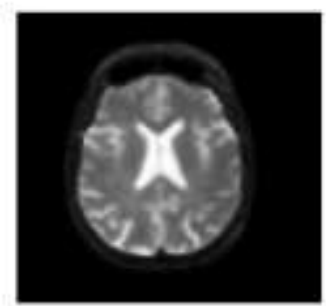

(c)

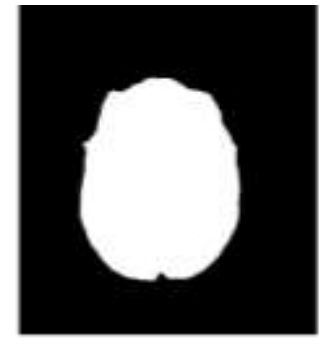

(e)

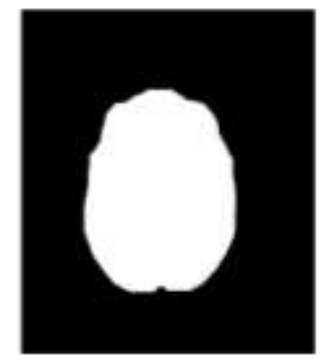

(g)

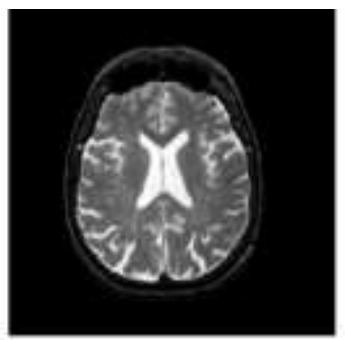

(b)

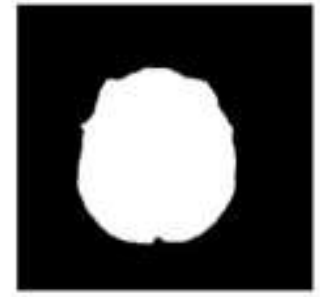

(d)

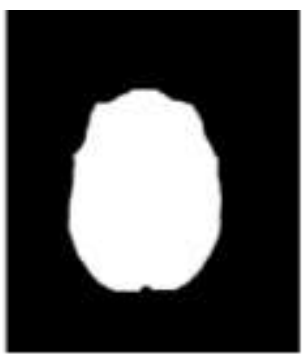

(f)

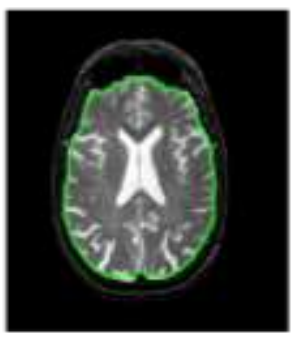

(h)

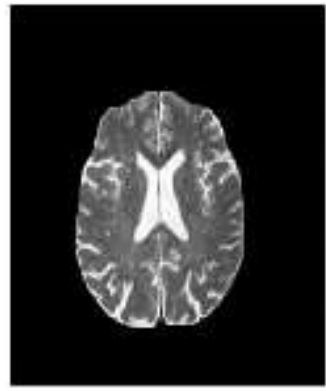

(i)

Figure 3. (a) Original brain MRI image, (b) Low Pass Filtered image, (c) Diffused image, (d) Binarized image, (e) Morphological Erosion, (f) Morphological Opening with Horizontal structuring element, (g)

Morphological Opening with Disk shaped structuring element, (h) Brain region in the main image, (i) Extracted brain region using 2D-BEA algorithm
The results in figure 3 shows that using 2D-BEA method the interested brain region is extracted and it removes the unwanted area like skull and other boundaries. It is fulfilling the main purpose of finding out the different possible locations of tumour for the later research.
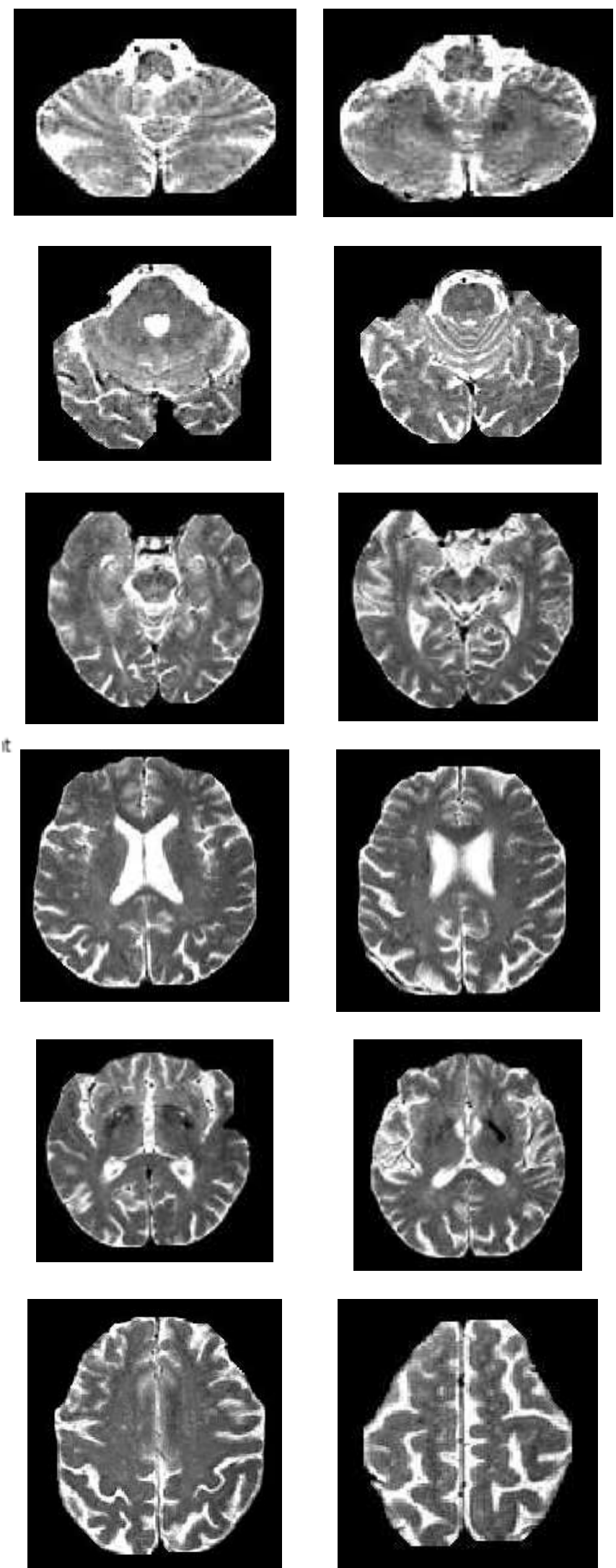

Figure 4. Extracted brain regions using 3D-BEA algorithm for same brain slices 


\section{Comparative analysis of developed algorithm with different segmentation techniques for MRI brain images}

In addition to the analysis of developed algorithm, the comparative analysis is also carried out with the existing algorithms. It would be easy to identify the efficient algorithm after this analysis. In this section total five segmentation algorithms have been implemented. Mainly cluster based algorithms are compared to validate developed algorithm. Below shows the algorithms and their steps with their importance in different aspects.

\section{A. $\quad$ Fuzzy c means based clustering}

One family of segmentation algorithms is based on the idea of clustering pixels with similar characteristics. Fuzzy cmeans has been shown to work well for clustering based segmentation (Benjamin, 2012), however due to its iterative nature this approach has excessive computational requirements. Below shows the algorithm of Fuzzy c means. Step 1: Initialize the membership Matrix

Step 2: Calculate the degree of membership

Step 3: Compute the centroid and update the new membership and Recalculate the degree of membership Step 4: If the difference of centroid matrix between new and previous iteration is less than the predefine value then recalculate the degree of membership.

\section{B. $\quad K$-means clustering}

It is one of the easiest methods of unsupervised learning algorithm that solve the well-known clustering issue (Funmilola and Adedeji, 2012). K-means is purposed by Macqueen in 1967. K-means is a simple clustering method which is having low computational complexity as compared to Fuzzy C-means. K-means clustering do not overlap the clusters.

$$
I=\sum_{i=1}^{e}\left(\sum_{k, X k \in G i} d_{\left(\mathrm{X}_{\mathrm{k}}-\mathrm{C}_{\mathrm{i}}\right)}\right)
$$

Below shows the algorithm of $\mathrm{k}$ means clustering algorithm for MRI brain image segmentation.

Step 1: Select K points as the initial centroids.

Step 2: Repeat

Step 3: Form K clusters by assigning all points to the closest centroid

Step 4: Re-compute the centroid of each cluster.

Step 5: Until the centroids don't change.

Simplicity and easy implementation are some advantages of k-means but it has several drawbacks as well. There is no standard for a good set of initial centres. Instead of random choices, initial k-means results can provide the initial points for the next run of the algorithm.
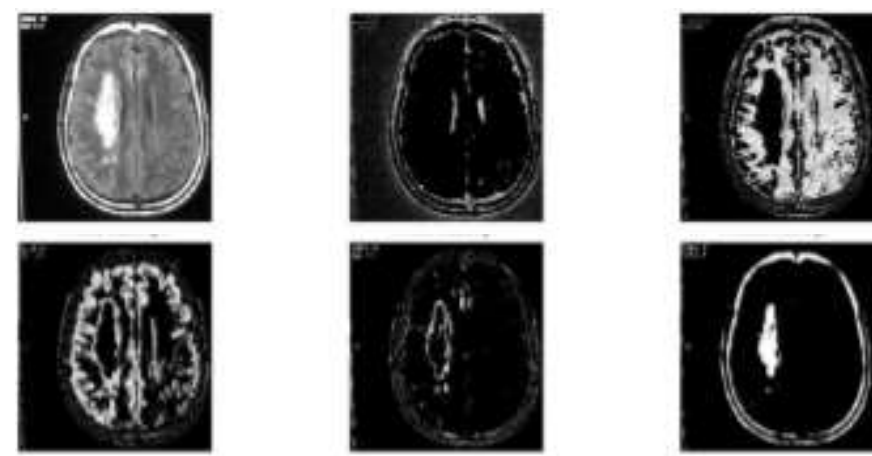

Figure 5. K means clustering based segmentation for 5 clusters

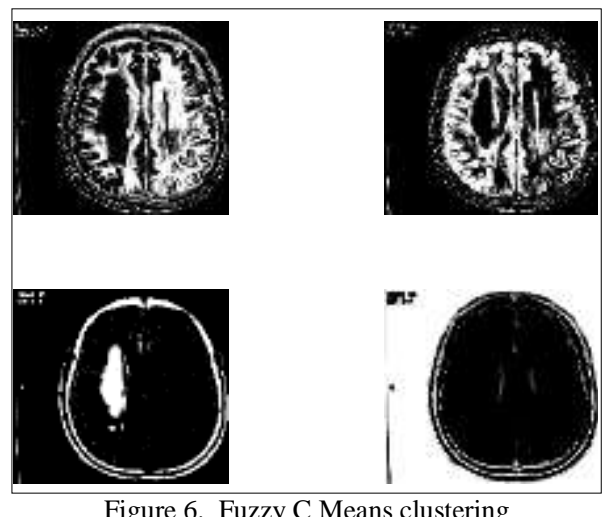

Below Table I depicts the importance of proposed algorithm 3D-BEA in all aspects towards better segmentation of brain MRI images. As compared to other segmentation algorithms searched by previous researchers the 3D-BEA algorithm is removing noise from the input image with limitations in computational time. Proposed algorithm is also efficient to identify the smooth edges as well as preserving information. This developed algorithm does not over segment the image so that blur effect comes down to null. Moreover it is so user friendly and automated that it does not expense time to diagnose unwanted area and only extract the desire region of brain MRI images.

Medical Resonance Image segmentation is very essential feature in most of image processing methods, which reflects anatomical structure of segment (brain tissue). The usefulness of these methods in clinical environment significantly depends on the ease of computation and the reduction of human intervention. The proposed method is based on histogram based gradient calculation, which segments out primary objects from T2 brain MR image (Gilanie and Attique, 2013). The applicability of this algorithm has been practically verified giving satisfactory results. It is established that the proposed method can be applied on other medical imaging modalities or other image processing domains and is quite efficient.

TABLE I. COMPARISON OF DIFFERENT IMAGE SEGMENTATION TECHNIQUES FOR MRI IMAGES OF BRAIN

\begin{tabular}{|c|c|c|}
\hline Parameter & Fuzzy C-Means & K-Means \\
\hline Noise & Cannot remove noise & Cannot remove noise. \\
\hline Smoothing & Doesn't smooth the image & $\begin{array}{c}\text { Different initial centroids will } \\
\text { bring about the different } \\
\text { results. }\end{array}$ \\
\hline
\end{tabular}


Proc. of the Intl. Conference on Advances in Information Processing and Communication Technology - IPCT 2016. Copyright (C) Institute of Research Engineers and Doctors. All rights reserved.

ISBN: 978-1-63248-099-6 doi: 10.15224/ 978-1-63248-099-6-27

\begin{tabular}{|c|c|c|}
\hline Separation & $\begin{array}{c}\text { Used for MRI image } \\
\text { segmentation }\end{array}$ & $\begin{array}{c}\text { Other region can also } \\
\text { separate. }\end{array}$ \\
\hline No. of Cluster & $\begin{array}{c}\text { Apriori Specification of } \\
\text { the number of cluster }\end{array}$ & $\begin{array}{c}\text { A problem of choice of } \\
\text { numbers of cluster N. }\end{array}$ \\
\hline $\begin{array}{c}\text { Over } \\
\text { segmentation }\end{array}$ & $\begin{array}{c}\text { Does not over segmented } \\
\text { image. }\end{array}$ & $\begin{array}{c}\text { Does not over segmented } \\
\text { image. }\end{array}$ \\
\hline $\begin{array}{c}\text { Time of } \\
\text { execution }\end{array}$ & 12.539335 seconds. & 14.116299 seconds. \\
\hline Parameter & $\begin{array}{c}\text { Brain Extraction } \\
\text { algorithm } \\
\text { 2D-BEA }\end{array}$ & $\begin{array}{c}\text { Brain Extraction algorithm } \\
\text { 3D-BEA }\end{array}$ \\
\hline Noise & $\begin{array}{c}\text { Removing the noise by } \\
\text { filtering. }\end{array}$ & $\begin{array}{c}\text { Removing the noise by } \\
\text { filtering. }\end{array}$ \\
\hline Smoothing & $\begin{array}{c}\text { More Smoothed image is } \\
\text { produced. }\end{array}$ & $\begin{array}{c}\text { More Smoothed image is } \\
\text { produced. }\end{array}$ \\
\hline Separation & $\begin{array}{c}\text { Can separate unwanted } \\
\text { area like skull but not } \\
\text { efficiently }\end{array}$ & $\begin{array}{c}\text { Can separate unwanted area } \\
\text { like skull as it is best for } \\
\text { Largest connecting } \\
\text { component identification }\end{array}$ \\
\hline No. of Cluster & $\begin{array}{c}\text { Does not require prior } \\
\text { knowledge of the number } \\
\text { of clusters. }\end{array}$ & $\begin{array}{c}\text { Does not require prior } \\
\text { knowledge of the number of } \\
\text { clusters. }\end{array}$ \\
\hline $\begin{array}{c}\text { Over } \\
\text { Time of } \\
\text { segmentation }\end{array}$ & $\begin{array}{c}\text { Does not over segmented } \\
\text { image. }\end{array}$ & $\begin{array}{c}\text { Does not over segmented } \\
\text { image. }\end{array}$ \\
\hline 8.732305 seconds & 76.019408 seconds. \\
\hline
\end{tabular}

\section{Conclusion}

Brain extraction algorithm is a general category of algorithms used to extract/evaluate features of a given brain scan. It also performs the very same task of post processing. In first stage, coarse brain is generated using filtering and thresholding. In second stage, morphological operations performed on binary image to segment the fine brain mask. It has been observed from the comparative analysis that existing methodology of segmentation is not able to remove noise as well as blur the images and moreover it does not extract brain area which is the main interest for further research and for finding out the tumour in the later stage. Results show the validity of the 3D-BEA algorithm and its advantages through comparison.

\section{Acknowledgment}

The authors would like to acknowledge the motivation and support given by radiologists and doctors from different MRI centers for providing the Brain MRI images and the corresponding information for the research.

\section{References}

[1] Ajala Funmilola A, Oke O.A, Adedeji T.O, Alade O.M, Adewusi E.A, " Fuzzy k-c-means Clustering Algorithm for Medical Image Segmentation", Journal of Information Engineering and Applications, ISSN 2224-5782 (print) ISSN 2225-0506 (online) Vol 2, No.6, 2012.

[2] Benjamin James Bush, “ Fuzzy Clustering Techniques: Fuzzy CMeans and Fuzzy Min-Max Clustering Neural Networks", SSIE 617 Term Paper, Fall 2012.

[3] Badredine Sayah, Bornia Tighiouart, "Brain tumour segmentation in MRI: knowledge-based system and region growing approach,", Internatinal journal of signal and imaging systems engineering, vol. 7, no. 2, pp. 65-74, 2014.

[4] Comaniciu, D.; Meer, P., "Mean shift: a robust approach toward feature space analysis," Pattern Analysis and Machine Intelligence, IEEE Transactions on pattern analysis and machine intelligence, vol.24, no.5, pp.603-619, May 2002.
[5] Cedric Wemmert, Anne Puissant, Germain Forestier, and Pierre Gancarski, "Multiresolution Remote Sensing Image Clustering," IEEE Geoscience And Remote Sensing Letters, vol. 6, no. 3,pp. 533-537, July 2009.

[6] Fritz albregtsen," region \& edge based segmentation",

[7] Ghulam Gilanie, Muhammad Attique, Hafeez-Ullah, Shahid Naweed, Ejaz Ahmed, Masroor Ikram, Object extraction from T2 weighted brain MR image using histogram based gradient calculation, Elsevier journal on Pattern Recognition Letters, Volume 34, Issue 12, 1 September 2013, Pages 1356-1363, ISSN 0167-8655,

[8] Jiawei Han, Vipin Kumar, "Clustering”, CIS 601 Fall 2004 Longin Jan Latecki. Website:

[9] K. Somasundaram, T. Kalaiselvi, Fully automatic brain extraction algorithm for axial T2-weighted magnetic resonance images, Elsevier journal on Computers in Biology and Medicine, Volume 40, Issue 10, October 2010, Pages 811-822, ISSN 0010-4825,

[10] M.S. Atkins, B.T. Mackiewich, Fully automatic segmentation of the brain in MRI, IEEE Transactions on Medical Imaging, Volume 17, Issue 1, February 1998, Pages 98-107.

[11] M.E. Brummer, R.M. Mersereau, R.L. Eisner, R.J. Lewine, Automatic detection of brain contours in MRI data sets, IEEE Transactions on Medical Imaging, Volume 12, Issue 2, June 1993, Pages 153-166

[12] P.Perona, J.Malik, Scale-space and edge detection using an isotropic diffusion, IEEE Transactions on Pattern Analysis and Machine Intelligence, Volume 12, Issue 7, July 1990, Pages 629-639

[13] Pilar Jarabo-Amores, Manuel Rosa-Zurera, David de la Mata-Moya, Raul Vicen-Bueno, and Saturnino Maldonado-Bascon, "SpatialRange Mean-Shift Filtering and Segmentation Applied to SAR Images.", IEEE Transactions On Instrumentation And Measurement, vol. 60, no. 2,pp. 584-597, February 2011.

[14] Raffaele Gaetano, Giuseppe Scarpa, And Giovanni Poggi, "Hierarchical Texture-Based Segmentation Of Multiresolution Remote-Sensing Images." IEEE Transactions on geoscience and remote sensing, vol. 47, no. 7, pp. 2129-2141, July 2009.

[15] Rafael C.Gonzalez, Richard E.Woods, "Digital Image Processing", Prentice Hall, Second Edition, 2007.Chapter 10, pp.567-634, ISBN 0201-18075-8.

[16] S.P.Raya, Low-level segmentation of 3-D magnetic resonance brain images--a rule based system, IEEE Transaction on Medical Imaging, Volume 9, Issue 3, September 1990, Pages 327-393

[17] Shaheera Rashwan, Mohamed Talaat Faheem, "A wavelet relational Fuzzy C-Means algorithm for 2D gel image segmentation", Computational and Mathematical methods in medicine, Volume 2013, Article ID 430516

[18] Wenbing Tao, Hai Jin, Yimin Zhang, "Colour Image Segmentation Based on Mean Shift and Normalized Cuts", IEEE Transactions on systems, man, and cybernetics-part b: cybernetics, vol. 37, no. 5, pp.1382-1389, October, 2007.

About Author :

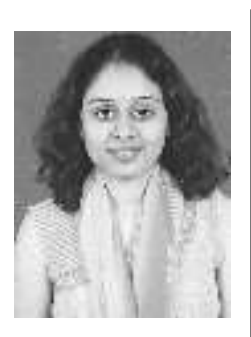

Vandana Shah received her master degree in VLSI technology from Nagpur University, India in 2012. She is currently a researcher in medical field for MRI brain tumour analysis and pursuing her $\mathrm{PhD}$ in Department of Electronics from the Nagpur university, her current research interest include, the computer assisted brain anatomy analysis and image segmentation techniques. 